\title{
Changes in diet following a cancer diagnosis in Scotland
}

\author{
L.F. Masson, D. Verra, H. Cumming, H. Cameron and E. Robinson \\ School of Pharmacy and Life Sciences, Robert Gordon University, Aberdeen, AB10 7GJ, UK.
}

Cancer survival has improved in Scotland from an age-standardised relative survival at five years of 39.6\% in $1987-1991$ to $59.3 \%$ in 2007-2011 in men and women aged 15-74 years ${ }^{(1)}$. However, long-term cancer survival is associated with increased incidence of diet-related diseases including coronary artery disease, osteoporosis, and diabetes ${ }^{(2)}$. This study aimed to assess diet and attitudes towards diet in cancer survivors in Scotland.

Eligible participants were men and women aged $\geq 18$ years who had received a diagnosis of cancer and used CLAN Cancer Support in Aberdeen. CLAN Cancer Support is a charity that supports individuals of any age who are, or have been, affected by cancer across North-East Scotland, Moray, Orkney and Shetland. Individuals were not eligible to participate if they were underweight (BMI $<18.5$ $\mathrm{kg} / \mathrm{m}^{2}$ ) or following specific dietary advice from their doctor or dietitian. Questionnaires were distributed in March 2015 and March 2016 to CLAN users by post (randomly selected), at organised events, and via CLAN volunteers. Participants returned questionnaires to CLAN by post or when visiting the facilities. The study was approved by the Robert Gordon University School of Pharmacy and Life Sciences Ethics Review Committee. Statistical analysis was carried out using SPSS version 21.

Seventy-four questionnaires were completed. The majority of participants were female (57\%), aged 55-74 years (74\%), and had received their first cancer diagnosis between 2009 and 2014 (55\%). The most common first cancer diagnosed was breast (34 \%) followed by prostate $(18 \%$ ) cancer. After their first cancer diagnosis, at least one quarter of participants reported reducing their frequency of consumption of red meat $(27 \%)$, processed meat $(37 \%)$, sweet biscuits and chocolate biscuits (30\%), buns, sweet pastries, scones, flapjacks, croissants and doughnuts (26\%), chocolates and chocolate bars (30\%), and crisps and other packet snacks $(28 \%)$. Most participants agreed that it was important for them to follow a healthy diet $(89 \%)$, and that they would consider improving their diet $(76 \%)$. There were no significant differences between sexes. Only $31 \%$ of participants reported that they received or obtained information about diet after their first cancer diagnosis; the most common sources of information were dietitian (14 \%), internet $(10 \%)$, information leaflets $(8 \%)$, and nutritionist and books (both $7 \%$ ).

Individuals may make positive changes to their diet following a cancer diagnosis and may be receptive to recommendations to further improve their diet. Further research is required to understand how best to deliver dietary advice to affect behavior change, and the role that local charities can make in supporting cancer survivors to make healthier dietary choices.

The authors acknowledge Dr Myra Mackenzie for reviewing and commenting on drafts of the questionnaire.

1. ISD Scotland (2015) Cancer Survival in Scotland 1987-2011. http://www.isdscotland.org (accessed September 2017).

2. Khan NF, Mant D, Carpenter L et al. (2011). Br J Cancer 106(Suppl 1), S29-37. 